\title{
Letteratura, potere ed etica. Laura Toppan dialoga con Gëzim Hajdari
}

Laura Toppan e Gezim Hajdari

\section{(2) OpenEdition}

1 Journals

\section{Edizione digitale}

URL: https://journals.openedition.org/cher/10806

DOI: 10.4000/cher.10806

ISSN: 2803-5992

\section{Editore}

Presses universitaires de Strasbourg

\section{Edizione cartacea}

Data di pubblicazione: 30 juin 2013

Paginazione: 27-46

ISBN: 978-2-35410-054-4

ISSN: 1968-035X

Notizia bibliografica digitale

Laura Toppan e Gezim Hajdari, «Letteratura, potere ed etica. Laura Toppan dialoga con Gëzim

Hajdari», reCHERches [Online], 10 | 2013, online dal 08 février 2022, consultato il 10 février 2022. URL: http://journals.openedition.org/cher/10806 ; DOl: https://doi.org/10.4000/cher.10806

\section{(c) (i) (ㅇ)}

Ce(tte) œuvre est mise à disposition selon les termes de la Licence Creative Commons Attribution Pas d'Utilisation Commerciale - Partage dans les Mêmes Conditions 4.0 International. 


\title{
Letteratura, potere ed etica. Laura Toppan dialoga con Gëzim Hajdari
}

\author{
LaURa Toppan \\ Université de Lorraine \\ GEZIM HAJdARI \\ Scrittore
}

Laura Toppan: Il titolo scelto per questo dialogo fa riferimento alla recente pubblicazione in Albania del volume Gjëmë. Genocidi i poezisë shipe ${ }^{1}$ (Epicedio. Il genocidio della poesia albanese) in cui Gëzim Hajdari parla del massacro perpetrato contro i poeti del suo paese durante la dittatura comunista. La repressione contro i rappresentanti della cultura albanese, cominciata in realtà fin dagli anni '20 sotto il regime feudale di Ahmet $\mathrm{Zog}^{2}$ e continuata durante l'occupazione nazi-fascista, riprese con sistematica ferocia nel periodo del Terrore rosso, cioè dalla fine della seconda guerra mondiale fino al 1989, l'anno che precede il crollo del regime di Enver Hoxha ${ }^{3}$.

1 G. Hajdari, Gjëmë: genocidi i poezise shipe, Tiranë, Shtëpia Botuese "Mësonjëtorja”, 2010.

2 Ahmet Lekë Bej Zog (noto come Zog I) nacque a Burrel nel 1895 e morì in Francia nel 1961. Fu Primo Ministro dell'Albania negli anni '22-'24, Presidente della Repubblica Albanese dal '25 al '28 e si autoproclamò Re d'Albania dal '28 al '39.

3 Enver Hoxha (1908-1985) nacque nel sud dell'Albania, nella città di Argirocastro. Fece i suoi studi nel liceo francese di Coriza e nel 1930, grazie ad una borsa di studio statale, frequentò l'università di Montpellier, che abbandonò molto presto. Dal '34 al '36 fu segretario al Consolato albanese di Bruxelles e nella capitale belga studiò legge all'università, ma anche qui non terminò gli studi. Ritornato in Albania divenne insegnante a Coriza e fu rimosso dal suo posto in seguito all'invasione italiana del '39 per essersi rifiutato di iscriversi al Partito Fascista Albanese. Con l'aiuto dei comunisti jugoslavi prese la guida del Partito Comunista Albanese (chiamato successivamente Partito del Lavoro), che andrà al potere il 29 novembre del 1944. Enver Hoxha si dichiarava un marxista-leninista ortodosso, grande ammiratore del dittatore sovietico Stalin prese: 
Gjëme è un libro che tenta di mettere insieme per la prima volta i frammenti della memoria collettiva albanese. Esso rappresenta «l'altra verità», quella scomoda, volutamente tenuta nascosta dai proclami della cultura «ufficiale» dell'epoca, che tutto ha messo in atto per cancellare le tracce di ciò che è accaduto nelle stanze del terrore di Stato. Sino ad oggi non esistevano saggi che percorressero cronologicamente la storia tragica della poesia, della letteratura e della cultura albanesi degli ultimi cinquant'anni. Si potevano trovare solamente alcune interviste, poche testimonianze o qualche libro che parlasse della terribile persecuzione messa in atto da Hoxha, ma non era ancora stato pubblicato un libro che racchiudesse le voci e i drammi di tutti coloro che, per mezzo secolo, furono costretti al silenzio dal regime e, per resistere, pagarono un prezzo altissimo. Attualmente, quello che sembra un semplice disinteresse da parte dei governi post-comunisti riguardo a coloro che, resistendo con la parola e con il sangue, si sono opposti alla cultura ufficiale, corrisponde in realtà ad una precisa volontà: non far emergere una verità storica estremamente pesante e compromettente. Questa è la ragione per cui sono passati già vent'anni dal crollo del regime e ancora non sono apparsi studi o saggi come Gjëmë. Genocidi i poezisë shipe.

Hajdari ha iniziato a raccogliere il materiale e a scrivere questo libro in Albania, precisamente in Darsìa - regione al nord del paese da cui proviene -, per continuare poi in Ciociarìa durante l'esilio italiano. Sono stati anni di ricerca, di studi, di letture, di approfondimenti, di riflessioni, di

come modello l'Unione Sovietica e irrigidì le relazioni con i suoi vecchi alleati in seguito alla condanna della loro ideologia decisa a Mosca nel '48. Temendo un'invasione da parte dell'Europa Occidentale, dal ' 50 Hoxha fece costruire in tutto il paese migliaia di bunker in cemento come posti di guardia e ricoveri di armi. La loro costruzione accelerò quando, nel '68, uscì ufficialmente dal Patto di Varsavia aumentando così il rischio di un attacco straniero. Intanto egli dichiarò trionfalmente che l'ateismo di stato era scritto nella Costituzione. A causa dell'isolamento del paese e del deperimento dei rapporti con il blocco sovietico, alcuni diritti civili come la libertà di parola, di religione, di stampa e di associazione (sebbene la costituzione del 1976 li enunciasse), vennero soppressi con una legge del '77 per garantire stabilità ed ordine. Nell'81 Hoxha ordinò l'arresto e l'esecuzione capitale di diversi dirigenti di partito e di governo accusati di corruzione e di attività controrivoluzionaria. Il suo regime dittatoriale fu durissimo e isolò l'Albania per decenni. Cf. Gabriel Jandot, L'Albanie d'Enver Hoxha (1944-1985), Parigi, L'Harmattan, 1994; Amik Kasoruho, Un incubo di mezzo secolo: l'Albania di Enver Hoxha, Lecce, Argo, 1994 (1a ed.), poi Nardò, Besa, 1998 (2a ed.); E. Frabetti, Le particolarità del comunismo di Hoxha, in «Futuribili», n. 2-3, 1997, p. 188; Edmond Tupja, Souvenirs d'un traducteur: j'étais l'interprète du tyran albanais Enver Hoxha, Parigi, l'Harmattan, 2001; Arshi Pipa, The Political Culture of Hoxha's Albania, in Tariq Ali (a cura di), The Stalinist Legacy. Its Impact on Twentieth Century World Politics, Londra, Penguin Books - Harmondsworth, 1984, p. 435-464. 
incontri con gli amici e i familiari delle vittime. Anni intensi e drammatici che, secondo l'autore, hanno segnato per sempre la sua esistenza. Non è stato facile dar corpo, volto, voce e nome ai poeti shqiptarë perseguitati, imprigionati, torturati, uccisi o morti di stenti nei lager e nei campi di internamento durante la dittatura di Hoxha, uno tra i regimi più feroci nella storia europea.

Le difficoltà incontrate da Hajdari nel reperire il materiale sono state enormi perché certe persone, responsabili di questi fatti tragici, ricoprono ancora cariche pubbliche nell'Albania post-comunista di oggi e utilizzano quindi qualsiasi mezzo pur di seppellire la memoria storica, evitando così che la verità venga resa pubblica, sia all'interno della nazione, che all'estero. In Albania comunque si continua a scavare e si continuano a trovare fosse comuni. I familiari attendono che il Parlamento di Tirana condanni i crimini commessi durante il Terrore di Stato e cercano i manoscritti dei loro cari, fatti sparire dalla repressione e dalla censura. L'Albania è un cimitero di corpi ed opere.

Gjëmë. Genocidi i poezisë shipe è un libro che evita di entrare in qualsiasi polemica ideologica, poiché l'unico scopo del suo autore è quello di tracciare le biografie dei poeti che si sono sacrificati in nome della libertà di parola e della giustizia e di dar loro voce attraverso i racconti dei superstiti o dei loro familiari e compagni. Tutto il materiale si basa su fonti documentate, che sono però sparse: il lavoro non finisce quindi con questo volume, poiché gli archivi di Stato non sono ancora stati aperti e tantissimi documenti non sono ancora stati consultati. È il Parlamento che deciderà quando farlo $\mathrm{e}$ poiché l'orrore da scoprire è inaudito, si cerca sempre di rinviare la data d'apertura. Hajdari sottolinea il fatto che sono trascorsi già vent'anni dalla fine del regime e nessuno ha ancora pensato di creare un luogo, un centro che raccolga le testimonianze del passato, per far conoscere alle nuove generazioni, che nulla sanno, ciò che è accaduto durante gli anni del terrore. Si è iniziato un lavoro di ricostruzione della memoria grazie a dei siti virtuali creati dai parenti delle vittime, ma non esiste un progetto statale. Per Hajdari, finché il Parlamento non dichiarerà il Terrore di Stato un crimine contro l'umanità e non sarà stabilita l'esattezza dei fatti, in Albania non ci potrà essere una vera democrazia. Un altro pericolo per la verità storica è il passare inesorabile del tempo, a causa del quale si teme la scomparsa dei testimoni diretti, di quei pochi che sono sopravvissuti ai lager, cioè coloro che sono la memoria vivente dei crimini commessi dalla dittatura. 
L'Epicedio di Hajdari è comunque uscito in Albania nell'agosto del 2010 presso Mësonjëtorja, una casa editrice di Tirana. Il direttore è stato molto coraggioso nell'autorizzarne la pubblicazione, poiché i rischi di rappresaglia nei suoi confronti e in quelli dell'autore sono elevati. Gjëmë. Genocidi $i$ poezisë shipe avrebbe potuto essere il libro dell'anno in Albania, invece è stato condannato al silenzio da quella che l'autore definisce «la mafia politica e culturale di Tirana». A conferma di tutto ciò, nella stampa albanese è uscita una sola recensione al volume, quella dello scrittore ed editorialista Ylli Polovina, nel quotidiano nazionale «Shqip». Questo dimostra che si è cercato di far passare sotto silenzio un lavoro che affronta verità dolorose e ancora scomode nell'Albania attuale. E non basta. Hajdari non solo non è stato invitato a presentare questo suo «compianto» alla Fiera del Libro 2010, ma da quando è in esilio non è mai stato invitato in madrepatria a parlare della sua opera, pur essendo tra i maggiori poeti albanesi viventi.

Per Hajdari sarebbe importante fondare un'università del Mediterraneo che si occupasse di erranza, del mito classico del viaggio, dell'interazione tra popoli e culture sulle opposte sponde del mar Adriatico, del bilinguismo, della tradizione orale, dell'esilio e delle letterature delle diaspore, per affrontare le sfide del futuro e per rifondare l'idea di «mediterraneità».

\section{Ripercorriamo ora alcune tappe significative della biografia di Hajdari.}

Egli nasce nel 1957 a Hajdarai, un piccolo villaggio sulle colline della provincia di Darsía, una regione al nord dell'Albania. Quelli sono anni in cui la letteratura albanese cerca di sopravvivere, sottraendosi ai diktat dell'ideologia socialista imposti dal partito. Hajdari inizia gli studi con Jozëf Radi - figlio del poeta perseguitato Läzer Radi - ed entra in contatto clandestinamente con la letteratura europea, in particolare con quella italiana. Inizia a comporre versi costruendo un linguaggio che si veste della drammaticità e della desolazione dell'ambiente che lo circonda, affronta le delusioni del momento storico sognando la fuga in un «altrove». La sua prima raccolta, Antologia della pioggia ${ }^{4}$, viene censurata, perché contiene

4 G. Hajdari, Antologia della pioggia/Antologjia e shiut, Sant'Arcangelo di Romagna, Fara, 2000. «E una silloge tra quelle più care al poeta: scritta negli anni Ottanta e consegnata all'editore N. Frasheri di Tirana nel 1985, non venne pubblicata. Secondo la censura del regime albanese il volumetto non era consono al cosiddetto realismo socialista che, come in nessun altro paese al mondo, ha mostruosamente sterilizzato per mezzo secolo la poesia e l'intera letteratura nazionale. La raccolta è uscita solo nel novembre del 1990, due mesi prima del crollo della dittatura comunista, subendo amputazioni. La ripubblicazione italiana include liriche scelte e alcuni testi inediti». 
degli elementi autobiografici che non corrispondono ai dettami della cultura ufficiale. La poesia di Hajdari diventa così atto di denuncia nei confronti del regime, poiché si rifiuta di cantare le lodi del «socialismo reale». Anche Erbamara ${ }^{5}$, la seconda plaquette di componimenti, verrà censurata ed entrambe le raccolte saranno pubblicate solo dopo la caduta del regime, nel 1990. Hajdari non smetterà di comporre versi anche in esilio, in Italia, dove vive ancora oggi e dove continua, secondo le sue stesse parole, a «scrivere in italiano e a tormentarsi in albanese, a scrivere in albanese e a tormentarsi in italiano».

Il suo corpus letterario comprende le raccolte seguenti, tutte in bilingue: Antologia della pioggia/Antologjia e shiut, Ombra di cane/Hije qeni', Sassi controvento/Gurë kundërerës ${ }^{7}$, Corpo presente/Trup i pranishëm ${ }^{8}$ (per la quale gli viene attribuito il premio Montale per la poesia inedita nel 1999) Stigmate/Vragë9, Spine nere/Gjëmba të zinji10, Poema dell'esilio/Poema e mërgimit ${ }^{11}$ (un lunghissimo poema epico «aperto» nel quale Hajdari denuncia tutti i crimini della vecchia nomenklatura e le menzogne di quella che è ora al potere), Maldiluna/Dhimbjehëne ${ }^{12}$, Peligòrga / Peligorga ${ }^{13}$ (del 2007). Nel 2008 è uscita l'antologia Poesie scelte ${ }^{14}$, che riunisce i più significativi componimenti selezionati tra le sue diverse raccolte.

Gëzim Hajdari: Farò parlare i miei versi per presentare il mio percorso di poeta migrante:

5 G. Hajdari, Erbamara/Barihidhur, Sant'Arcangelo, Fara, 2001.

6 ID., Ombra di cane / Hije qeni, Frosinone, Dismisuratesti, 1993.

7 ID., Sassi contro vento/Gurë kundërerës, Milano, Laboratorio delle Arti, 1995.

8 ID., Corpo presente/Trup i pranishëm, Botimet Dritëro, Tiranë, 1999 (1a ed.); Nardò, Besa 2011 (2a ed.).

9 ID., Stigmate/Vragë, Nardò, Besa, 2002.

10 ID., Spine nere/Gjëmba të zinj, Nardò, Besa, 2004.

11 ID., Poema dell'esilio / Poema e mërgimit, Sant'Arcangelo, Fara, 2000.

12 ID., Maldiluna/Dhimbjehënei, Nardò, Besa, 2005.

13 ID., Erbamara/Barihidhur, Sant'Arcangelo, Fara, 2001.

14 ID., Poesie scelte, Edizioni “Controluce”, 2008 (ed. italiana); Poezi të zgjedura, Nardò, Besa, 2008 (ed. albanese). 
Un verso cieco

senza memoria

è il mio corpo,

nato in un paese povero.

(da Ombra di cane)
Con le mie notti nate dai tuoi giorni giungerò alle tue secche labbra, io sopravvissuto delle dittature oblìo di tutte le libertà;

busserò a te come ad una città santa, proibita agli infedeli.

(da Stigmate)
Canto il mio corpo presente nato da questo freddo spazio che nulla promette.

Di notte,

visioni di bianchi templi mi richiamano nel vuoto.

Ho sognato campi solitari per cercare i segni confusi e capire la maschera dei cieli che ama gli abissi.

Non so perché guardo a lungo la linea sottile dell'orizzonte o le cime brulle con uccelli neri.

Dove si nasconde ciò che non trovo sulle tremule alghe

o nei licheni bianchi?

Procedo nel verde consumato e non porto nulla oltre il mio corpo.

Non lascerò nulla

(da Corpo presente)
Com'è triste Roma

senza di te amore mio, senza i tuoi occhi, le tue labbra (rosse di sangue), la tua ombra.

Accanto a me sei come una collina, campo di grano o bosco vergine dove bussano la piogggia e il mondo.

Se tu chiami ti rispondono gli angeli, se tu gridi ti sente il mare, se tu piangi ti accolgono le rovine.

Ti perdo e ti ritrovo tra mura e grotte viva e uccisa dalle stesse pietre, dalle stesse ombre (da Corpo presente) 
Sono la verità

di un viaggio e di una linea d'Ombra custoditi sulla terra viva e chiusa che vuole nasconderci qualcosa.

Vivo sospeso

senza appartenere a nessuna dimora, al bivio di ogni equilibrio.

Ho camminato con passo lento

fra i morti assetati, per raggiungere l'alba dell'indomani di incendi e tregue.

Infinito che mi ospiti, sono stanco del Tempo e del vuoto.

Cos'è il mio frammento o il tuo frammento?

La mia angoscia diventa orizzontale come la mia illusione, sottile diventa anche il muro che mi difende e mi divide (da Corpo presente) 
L. T.: Nel 1991 Hajdari è tra i fondatori del Partito Repubblicano Albanese e del Partito Democratico della città di Lushjne, oppositori del regime in carica. Nello stesso anno fonda il settimanale «Ora e Fijales» ( Il momento della Parola») di cui è vice-direttore e scrive regolarmente sul quotidiano nazionale «La Repubblica» denunciando i crimini, gli abusi, le speculazioni della vecchia dittatura di Hoxha e dei recenti regimi post-comunisti; si candida inoltre al Parlamento. Nel 1992, a causa delle sue scelte intellettuali e politiche, è minacciato di morte e sfugge a un attentato perpetrato presso la sede del Partito Repubblicano. Decide allora di abbandonare l'Albania e, per la sua vicinanza geografica, sceglie l'Italia come paese dell'esilio. È Frosinone la città che lo accoglie, ove trova una vasta comunità di connazionali e dove vive tuttora come "cittadino onorario per meriti letterari". Con questo intervento Hajdari vuole presentare, per la prima volta in Francia, Gjëmë. Genocidi i poezisë shipe per testimoniare come uno scrittore migrante abbia anche un dovere etico rispetto alla propria opera.

G. H.: Questo libro non è un saggio accademico, ma un Compianto Funebre che racconta del massacro compiuto contro i poeti albanesi in un arco di tempo che va dal 1920 al 1989, anno in cui, per l'ultima volta, venne trucidato un poeta albanese. Il suo corpo rimase esposto per tre giorni sulla piazza della città di Tirana come monito ed esempio di ciò che sarebbe potuto capitare a chi avesse osato sfidare l'ideologia di Stato. Epicedio è un libro che, per la prima volta nelle vicende dell'Albania contemporanea, tenta di mettere insieme i frammenti della memoria collettiva, storica e culturale del paese. Un quotidiano molto importante di Tirana, «Shqip», ha recensito il mio lavoro, ma c'è una certa reticenza nello scoprire questi orrori, poiché il confronto con la verità che emerge fa male e perché, ancora oggi, coloro che governano il paese e che gestiscono la vita pubblica e culturale sono gli stessi di ieri, riciclati con nuove "maschere". Questo libro le fa cadere, scoprendone i veri volti. È un'opera scritta non per sollevare e alimentare polemiche, ma per raccontare, attraverso le testimonianze rilasciate dai familiari delle vittime, attraverso le interviste ai superstiti, le letture, gli approfondimenti e i documenti inediti, ciò che in Albania è veramente accaduto.

L. T.: Il libro è frutto di una ricerca durata sette anni - iniziata nel 2002, in Darsìa, e terminata nel 2009 in Ciociaria - che però continua. È in qualche modo un libro aperto, che si arricchisce via via di nuovi apporti e scoperte poiché la verità sta venendo a galla poco a poco. La variante italiana che uscirà alla fine del 2012 - ha già circa cento pagine in più rispetto 
all'edizione albanese, mentre in Germania è uscita in versione ridotta nella rivista "Matrix» alla fine del 2009. Anche in Francia alcuni editori hanno intuito l'importanza del lavoro e hanno dimostrato per esso il loro interesse, ma per il momento hanno dato una risposta negativa quando sono venuti a conoscenza dei fatti riguardanti lo scrittore Ismail Kadaré15, che è il massimo riferimento mondiale per la letteratura albanese. Nel suo libro Hajdari ricostruisce passo dopo passo la controversa figura dello scrittore, facendo chiarezza sul ruolo che egli ebbe durante la dittatura di Hoxha. Hajdari dimostra come l'immagine del rifugiato politico, che Kadaré ha pazientemente costruito negli anni intorno alla propria persona, sia in realtà un mito, e non corrisponda assolutamente a verità. Nello studio di Hajdari il Kadaré attivo durante la dittatura risulta un uomo senza principi e di basso profilo morale, che non soltanto tollerò il regime di cui fu connivente, ma lo sostenne attivamente, sia come scrittore sia come funzionario di stato. Kadaré dichiara di "esser fuggito dall'Albania" nel 1990 e di aver scelto Parigi per quello che egli definisce "il proprio esilio" chiedendo l'asilo politico: in realtà egli, in patria, faceva parte dello stretto entourage di Hoxha. La sua, quindi, è una figura molto ambigua nello scenario culturale europeo, tanto che molte volte è stato candidato al Nobel per i suoi meriti letterari, ma mai il premio gli è stato attribuito, forse proprio a causa del suo passato compromettente. La critica palese al ruolo svolto da Kadaré nell'Albania di Hoxha non costituisce certo il tema principale di Epicedio albanese. Hajdari dedica il proprio lavoro soprattutto alla memoria di tutte le vittime del regime: tutti quegli uomini e quelle donne che persero la vita per una parola sbagliata, per un verso attraverso cui cercavano di affermare l'umanità in tempi oscuri.

15 Ismail Kadaré è nato nel 1936 ad Argirocastro, città dell'Albania meridionale. Si è laureato in Storia e Filologia presso l'Università di Tirana e ha studiato per due anni letteratura all'Istituto Gorki a Mosca, che ha dovuto abbandonare a causa del complicato rapporto diplomatico tra Albania e Unione Sovietica. Tornato in patria inizia la sua carriera come giornalista presso la rivista «Drita» e, poco a poco, diventa conosciuto e gli affidano la direzione della collana Les lettres albanaises. Inizialmente si dedica alla poesia, passione che aveva sin dall'adolescenza, e pubblica varie raccolte che hanno successo. Nel '63 esce il suo primo romanzo, Il generale dell'armata morta, a partire dal quale decide di dedicarsi quasi esclusivamente alla prosa. Nel '90 chiede ed ottiene l'asilo politico in Francia. Nel 1993 vince il Premio Mediterraneo con il romanzo La Pyramide. Nel 2005 gli viene attribuita la 1a edizione dell'International Booker Prize e nel 2009 il premio Principe delle Asturie per la letteratura. Nello stesso anno gli viene conferita la Laurea Honoris Causa in Scienze della Comunicazione Sociale e Istituzionale dall'Università di Palermo, voluta caldamente dagli arbërësh della Piana degli Albanesi. È stato quattro volte candidato alla selezione finale per il Premio Nobel ed è membro d'onore dell'Académie Française. 
G. H.: Epicedio albanese racconta gli orrori di coloro che si sono sacrificati per la libertà di parola e per i diritti umani, ma riporta anche i versi di quelli che invece inneggiarono alla dittatura, che dedicarono migliaia di pagine al tiranno, al suo partito, alla lotta di classe. Ecco perchè la pubblicazione di questo libro in Francia creerebbe forse degli sconvolgimenti e toccherebbe anche qualche interesse economico... Lo stesso è accaduto in Italia: all'inizio il mio editore si è spaventato, ma ora che il saggio è uscito in Albania si è tranquillizzato ed è disponibile alla pubblicazione. Ora spero che Epicedio venga pubblicato in altri paesi poiché contiene delle verità inaudite che non possono più essere ignorate. Il libro riporta le accuse folli e assurde fatte agli intellettuali e ai poeti albanesi, i processi sommari, le torture nelle carceri, i nomi dei boia, dei procuratori, dei giudici; fa anche riferimento al massacro dei poeti e dei dissidenti rumeni, polacchi e sovietici che avveniva negli stessi anni. Ciò che accadde in Russia e in Cina durante la rivoluzione culturale, nonché negli altri paesi del blocco sovietico, lo si sa, ma l'opinione pubblica ignora, per gran parte, quello che avvenne negli stessi anni in Albania. Per scrivere questo saggio ho dovuto leggere centinaia di libri e di articoli, incontrare ed intervistare le famiglie dei perseguitati, custodire le fotocopie di alcuni documenti preziosi e segreti arrivati da Tirana. Alcuni familiari delle vittime sono infatti riusciti a farsi assumere presso gli Archivi di Stato - che non sono ancora stati riaperti - con l'unico scopo di metter mano su documenti importanti e fotocopiarli affinché la verità inizi a venire a galla. Così in Epicedio c'è una ricchissima bibliografia e prima di cominciare a scrivere ho consultato un avvocato per capire come potermi muovere. Poi si è presentato il problema della forma e poiché tutta la mia opera è concepita come un poema epico ho scelto quella del compianto funebre: Epicedio si aggiunge quindi, idealmente, al mio corpus epico.

Appartengo a una stirpe che proviene dalle montagne del nord del paese, una stirpe che durante la lunga occupazione turca (nel $1479 \mathrm{i}$ turchi conquistarono definitivamente l'Albania) si è autogovernata per 500 anni tramite il Kanún, un codice giuridico orale costituito da leggi consuetudinarie che 'regolano' i comportamenti in materia di ospitalità, di diritti matrimoniali e addirittura all'interno delle faide familiari. Poteva capitare, ad esempio, che un guerriero si distinguesse in battaglia per coraggio, forza, amore per la patria e che prima di seppellirlo le donne inneggiassero alle sue qualità cantando una sorta di compianto funebre: «Tu che non hai mai indietreggiato davanti alle difficoltà/ tu che hai amato la patria/ tu che ti sei sacrificato in nome delle donne del nostro popolo / tu che hai dato la vita per le nostre genti e le nostre 
terre», e così via. È importante precisare che l'Albania non ha mai attaccato un altro paese, ma ha sempre combattuto guerre difensive ed è stata occupata per 800 anni dai romani, 500 dai turchi, successivamente dai francesi, dagli austriaci, dai bulgari, dai serbi, dagli italiani. Così, per parlare dei crimini di Stato, ho scelto questa forma di compianto che enumera il coraggio e le virtù eroiche dei poeti albanesi. Tanti avevano studiato all'estero, nelle università francesi, tedesche, italiane, austriache e avrebbero dovuto essere i padri fondatori della nazione albanese, invece sono stati tutti massacrati poiché lo scopo del regime di Hoxha era quello di cancellare questi legami tradizionali dell'Albania con il resto dell'Europa. Epicedio parla di questo "genocidio".

L. T.: Ricordiamo che l'11 maggio 2010 si è inaugurata a Tirana, presso il Museo Nazionale, una mostra fotografica dedicata alle vittime di Enver Hoxha. Inoltre il 20 luglio 2010 è stato costituito l'Istituto dei Crimini del Comunismo che dovrà indagare e far luce sui crimini perpetrati sotto la sua dittatura.

G. H.: La mia volontà è di ristabilire la verità storica, poiché il problema è che alcuni intellettuali, tra cui Ismail Kadaré, si spacciano per difensori dei diritti umani e simboli della letteratura albanese, ma la realtà è molto diversa. Ad esempio, proprio Kadaré ha dedicato i poemi più celebri al Partito di Tirana ed è stato deputato nel Parlamento Albanese, quindi ha avuto un ruolo attivo nell'approvazione delle leggi che hanno fatto fucilare più di 150 poeti e hanno mandato nei lager migliaia di uomini e donne albanesi. Inoltre, egli ha dedicato più di 1200 pagine di prosa (è tutto documentato) al Tiranno. Questi fatti non vengono né raccontati, né pubblicati all'estero e quindi con Epicedio cerco di riportare le storie di vita di tutti i poeti morti per la libertà di Parola, senza appartenenza politica. È un libro molto doloroso: scrivendolo ho sofferto atrocemente, mi ha sconvolto e ha cambiato la mia esistenza. Non si sa ancora tutta la verità, poichè non c'è ancora una legge in Albania che condanni i crimini commessi e che porti all'apertura dell'archivio di Stato; non sappiamo ciò che accadde nelle stanze del Sigurimi, la polizia segreta albanese. Questo è il grande problema.

L.T.: Come e dove vivono ora i sopravvissuti ai lager e ai campi di internamento?

G. H.: La maggior parte di essi vive in Occidente: in Belgio, in Italia, in Francia, in Germania, in Grecia, in Inghilterra e molti anche negli Stati Uniti. 
Una volta crollato il muro di Berlino sono dovuti scappare e fuggire il più lontano possibile dalla madrepatria, diventata come una Medea che divora i propri figli. Se in Germania Hitler dichiarò guerra agli Ebrei, Hoxha dichiarò guerra al suo popolo! La mia missione è quella di ricostruire la memoria storica del mio paese e poiché molti politici non vogliono che si scopra la verità sono stato condannato al silenzio. Nell'arco di vent'anni di esilio e di lavoro letterario non sono mai stato invitato in Albania, poiché considerato un autore scomodo. La definizione che danno di me è quella di "nemico dell'Albania", anche perché scrivo in bilingue e quindi anche in italiano, nella lingua di coloro che occuparono l'Albania, i fascisti di Mussolini. Pur promuovendo da due decenni l'immagine della cultura albanese in Italia e viceversa, - ho infatti tradotto in albanese molti poeti italiani, come ad esempio Dario Bellezza e Luigi Manzi, quindi ricoprendo il ruolo di colui che cerca di recuperare il legame storico fra le due coste (l'Italia e l'Albania hanno infatti condiviso, per un certo periodo, lo stesso destino) - la mia voce, nella madrepatria, è muta.

L. T.: Propongo ora all'autore di leggere qualche pagina di Epicedio albanese per dare un'idea al lettore dello stile, del tono e del contenuto dell'opera.

G. H.: Petrit A.Velaj, ex-perseguitato politico e compagno di cella del poeta Kudret Kokoshi, racconta: «Sono trascorsi più di sessant'anni da quando ho lasciato la terribile prigione di Burrell dove ho visto morire per le torture decine di compagni di cella. Ricordo che una sera alcuni detenuti ebbero un brutto presentimento, come se dovesse accadere qualcosa. Esat Sherifi, di Gjormi, e Rexhep Sulo, di Berat, decisero di intonare un canto disperato, le cui parole suonavano così: «Albania, madre nostra, / nuvole nere ti coprono, / i tuoi figli d'argento / ad uno ad uno mangiati dalle serpi! ». Le parole fecero raccogliere intorno a loro tutti i compagni del carcere e le conseguenze furono tragiche. Infatti qualche giorno dopo le guardie chiamarono nel cortile della prigione tutti noi detenuti e ci dissero che il questore della città sarebbe venuto a parlarci. Faceva molto freddo. Una volta giunto, il questore B. Goxho, con tono solenne, estrasse dalla tasca della giacca un foglio e disse: «Chi sentirà pronunciare il proprio nome sarà condannato a morte!». Secondo la prassi del carcere cinquanta dei nostri compagni dovevano essere prima torturati, poi fucilati. Dopo la lettura del macabro elenco le guardie ci risero in faccia dicendoci: «E finita per voi!». E Velaj continua: «Una mattina i boia entrarono nella cella di Avdul Hasim Kokoshi e di Gjergji Kokoshi, quest'ultimo ex-Ministro della Pubblica 
Istruzione. Il prigioniero venne appeso a testa in giù e poi iniziarono torture disumane. I secondini colpivano senza pietà con tutti i mezzi a disposizione. L'orribile sorte toccò per prima ad Avdul Hasim, che resistette da vero uomo a dolori insopportabili. Mentre i boia triplicavano gli atroci colpi, all'ingresso della cella due poliziotti iniziavano ad intonare dei canti epici accompagnati da strumenti tradizionali. Una volta - continua il suo macabro racconto A. Petrit Velaj - io e i poeti Kudret Kokoshi e Patër Mëshkalla stavamo andando a seppellire i resti di un nostro compagno morto per le torture. Camminavamo in silenzio, portando nel cuore un dolore immenso per il destino dei nostri cari amici e per quel che ci aspettava nel carcere tra i più terribili d'Europa. Dietro di noi i secondini, che cantavano un ritmo perverso e danzavano per schernirci: "Chi bacerà la morte domani?/ Ovviamente Kudret e Petrit/ e Don Mëshkalla».

Il poeta Don Patër Mëshkalla, oltre che teologo, filosofo e grande polemista, fu uno dei redattori di «Leka», rinomata rivista dei gesuiti di Shkodër (Scutari). Venne ammanettato dai poliziotti il 29 aprile del 1967 durante una riunione presso l'Istituto "L. Gurakuqi" di Skodër. Come pretesto per il suo arresto ci si servì della lettera che egli aveva spedito al Premier Mehmet Shehu in cui accusava il suo governo di essere responsabile del genocidio spirituale del suo popolo. Mëshkalla protestò contro le false accuse nei suoi confronti, ma il Sigurimi lo picchiò a sangue con calci e con i fucili nonostante fosse un anziano ultrasettantenne. Tre mesi dopo, in un luogo segreto, venne processato e condannato - per la seconda volta - a dieci anni di carcere, con l'accusa di "agitazione e propaganda contro il potere del proletariato". Ne aveva già subiti quindici a Burrell, prigione sulla quale circolava il detto: "Chi entra a Burrell, più non esce!" In questa prigionefortezza, situata ad 80 chilometri a nord di Tirana, i detenuti politici non svolgevano lavori forzati come a Spaç, ma venivano messi in isolamento per tutti gli anni della pena». E Petrit A. Velaj continua: «Quando arrivammo a Qershia e Burrelit, collina dove venivano seppelliti i detenuti, trovammo il corpo di un nostro compagno riesumato il giorno prima dalla fossa. Era stato fatto a brandelli anche dagli animali notturni, oltre che dalle torture. Le fosse, secondo l'ordine della direzione carceraria, non dovevano essere scavate a più di $50 \mathrm{~cm}$. di profondità: dal 1947 al 1948 abbiamo seppellito 720 prigionieri politici morti sotto le torture», aggiunge il poeta Velaj, sopravvissuto alle galere di Spaç, dove venivano rinchiusi gli intellettuali albanesi più "pericolosi" per il regime. 
L. T.: Diamo un altro esempio di testimonianza dell'Epicedio albanese. Hajdari ha conosciuto Jozëf Radi, figlio del poeta perseguitato Läzer Radi e di lui racconta: «Il poeta Lazër Radi (1916-1998) fu messo alla berlina nel campo di internamento di Savër, a Lushnje, la mia città - dove si trovavano ben 11 campi destinati a internare i "nemici" di Stato. È accaduto il 20 ottobre 1982, al Palazzo della Cultura, in presenza di 600 persone al seguito del segretario di partito Petraq Nushi: lo hanno insultato, gli hanno sputato addosso e tirato dei sassi, ma lui, immobile come una statua, non ha mosso ciglio, sfidando le pietre con le sue parole. Tra le mani stringevo la mia pietra colma di rabbia. Si è alzato un collaboratore della dittatura, Rustem Bega, gridando: «Lazër Radi, vogliamo sapere perché continui a parlare male del comunismo? Che male ti ha fatto il potere del proletariato?» Fiero e coraggioso Lazër, uno dei più grandi intellettuali della nazione albanese, ha risposto: «Mi ha condannato a sedici anni di carcere e a trenta di internamento in un campo [...]».

Lazër Radi nasce a Prizren, in Kosovo. A causa delle violenze subite da parte dei serbi e dei montenegrini la sua famiglia abbandona il Kosovo nel '29 e parte in esilio in Albania. Lazër termina le scuole medie a Tirana e vive tra la capitale e la città di Shkodër, centro di grande fervore culturale. Con i suoi compagni partecipa alla fondazione della società culturale «Besa Shqiptare». Durante il liceo fa l'attore di teatro. In quegli anni in Albania nascono i primi gruppi d'ispirazione comunista e Radi entra in contatto con le nuove idee che iniziano a circolare. Egli prende le distanze dagli ideali della rivoluzione bolscevica nel momento in cui i serbi e i montenegrini incominciano a commettere violenze nei confronti dei cittadini innocenti ed inermi del Kosovo.

L'incontro con il poeta Migjeni, nell'estate del '36, lascia un segno profondo nella vita di Radi e sono di quel periodo i suoi primi articoli sulla stampa dell'epoca. Nel ' 38 termina le scuole superiori, a Shkodër, e parte poi per Roma per studiare all'università "La Sapienza". Nella città eterna inizia per lui una nuova vita, ma nell'aprile del '39 viene espulso temporaneamente dall'Italia in seguito all'invasione dell'Albania da parte di Mussolini. Si laurea nel ' 42 in giurisprudenza con il massimo dei voti e il professor Vito Cesarini Sforza lo vorrebbe come suo assistente all'università, ma il giovane kosovaro preferisce tornare in Albania per servire la causa del proprio popolo. Il rientro in patria segnerà il suo calvario e quello della sua famiglia. Infatti nel '44 Lazër viene arrestato e nel ' 45 condannato a trent'anni di carcere accusato di esser stato "un anticomunista reazionario al servizio degli italiani". Dopo aver scontato dieci anni di lavori forzati viene "liberato" 
e subito dopo spedito nei campi di internamento, dove rimarrà fino al ' 74 . Mentre si trova in prigione il Sigurimi arresta e condanna a diversi anni di carcere anche la moglie Viktoria. Dopo averla torturata, per poterla condannare la polizia di stato troverà un pretesto: viene accusata di essere un agente segreto al servizio di Tito! Così il poeta e la sua famiglia trascorrono la loro vita lavorando nelle paludi di Savër, di Shtyllas, di Radostinë, di Kuç, di Çermë e poi di nuovo di Savër affrontando lavori massacranti nei campi piena di melma e colpiti dalla malaria. Radi potrà ritornare a Tirana solo nel 1991. Il suo calvario durò quarantasei anni: iniziò all'età di ventotto anni e terminò quando ne aveva 75».

G.H.: Il processo di Trifon Xhagjika (1923-1963) sconvolse l'opinione pubblica albanese. Egli trascorse l'infanzia nel Peshtan, un paesino vicino alla città di Tepelena, e all'età di 8 anni vide bruciare la propria casa natale (erano gli anni della guerra italo-greca). Egli termina le scuole superiori a Elbasan e chiede una borsa di studio per andare a studiare lettere a Mosca, ma la sua domanda viene respinta. Agli inizi degli anni '50 Trifon si laurea in Scienze Militari a Tirana ed inizia a lavorare nell'esercito come ufficiale di artiglieria. Più tardi entra nel Ministero della Difesa come giornalista presso il periodico «Luftëtari» (Il combattente). La letteratura è la sua passione e pubblica dei testi sui periodici nazionali «Drita», «Nëntori» e «Ylli». La sua prima raccolta, intitolata Gjurmëti (Le orme) esce nel '59. Egli apparteneva a quella generazione che aveva sognato un'Albania diversa, non quella della dittatura comunista, così ben presto il giovane poeta soffre della mancanza di libertà nel Paese e non ha paura di fare dichiarazioni esplicite contro il regime.

La poesia Aiutatemi, non riesco a ridere suonò come una critica per la censura. Parole crude, che denunciavano apertamente la dittatura: «Non posso,/ non posso,/ non posso./ Ho visto la Patria nuda,/ senza amici e compagni/ [...]/ l'ho presa per mano/ per farla crescere nel mio cuore [...]/ Fratelli -/ Se cercate la Patria,/ ce l'ho io./ Aiutatemi, non riesco a ridere, / Aiutatemi per farmi gioire./ La Patria è nuda' ${ }^{16}$ ».

Trifon dovette subire anche la separazione dalla moglie di origine russa. Negli anni '60, infatti, l'Albania interruppe i rapporti con l'Unione Sovietica, così per legge tutti coloro che avevano sposato donne russe dovevano divorziare. Il giorno in cui Trifon accompagnò la moglie e la figlia all'aereoporto

16 Afrim Imaj, Poeti i pushkatura Trifon Xhagjika në rrëfimin e të motrës, Sotira Tole, in «Panorama», Tiranë, 23.5.2006. 
promise loro che presto sarebbero stati di nuovo insieme. Non si dava pace. Tentò di fuggire all'estero attraversando il lago di Ohrid, ma venne arrestato e condannato insieme ai suoi compagni di fuga: Stavri Rafeli e suo fratello, Ivan Cani, nonché i due noti giornalisti Fadil Kokamani e Vangjel Lezho. Uomo dai capelli color paglia, di poche parole, Trifon venne ammanettato dai guardiani "rossi" in un caldissimo giorno di agosto mentre stava per uscire dalla casa della sua amata sorella Santa. Una scena commovente. Decine di poliziotti lo fermarono dicendogli: "Trifon Xhagjika, in nome del popolo, sei in arresto!" L'altra sorella, Sotira, racconta: "Trifon sapeva che sarebbe stato arrestato e quel giorno, diversamente dagli altri, non accettò che Santa lo accompagnasse sino al cancello. Dopo il suo arresto ci riunimmo tutti, sorelle e fratelli. Ci sentivamo come se una montagna fosse crollata su di noi. Non sapevamo la causa dell'arresto di Trifon, ma nostro fratello Tasho lo collegava ai testi che Trifon gli aveva confidato affinché li nascondesse a casa sua ${ }^{17} »$.

Era proprio così.

«Nel 1963, insieme a Trifon, - continua Sotira - vennero arrestati e condannati alla fucilazione un gruppo di giovani. Mio fratello venne accusato di "agitazione e propaganda" contro il potere del popolo e i suoi testi furono definiti testi "reazionari". Egli distribuiva le sue poesie clandestinamente ad amici e conoscenti e i suoi versi "blasfemi" venivano accolti con entusiasmo dai lettori: "Misera te, mia Repubblica/ Ti infanghi davanti ai signori/ Che spengono la tua luce nel buio/ Per farla accendere di giorno/ Ti offrono il pane da mangiare/ Ma ti tagliano le mani/ Ti portano alla sorgentel Ma ti chiudono la bocca ${ }^{18}$ ".

Le sue poesie sfidarono pubblicamente la dittatura. Ecco cosa scrive nel componimento dal titolo L'apoteosi dei nostri giorni: «Trappole/ e menzogne/ passeggiano libere per le strade./ Noi le conosciamo,/ ma abbiamo paura di toccarle,/ abbiamo paura di fermarle,/ vengono difese dalle guardie blindate ${ }^{19}$. Sono versi che il poeta stesso difese pagando con la propria vita: infatti Trifon Xhagjika venne condannato a morte. Il processo, senza difesa - perché nell'Albania di Enver Hoxha non era ammessa - venne fatto presso il Tribunale di Tirana. Le sue parole, dopo la sentenza, lasciarono sbigottiti i giudici della corte. Il ribelle, una volta condannato, invece di chiedere pietà

17 Ibidem.

18 Ibidem.

19 Ibidem. 
per la sua vita, coraggiosamente rispose agli inquisitori: «Datemi un cannone per colpire il vostro regime!».

Durante il processo Trifon osò declamare per l'ultima volta i suoi versi: «Nessuno può fermare la mia furia./ No!/ Non posso perdonarti./ La mia lingua si è sciolta./ Uccidimi se vuoi./ Nelle tue notti insanguinate/ ascolterai la mia voce:/ Ti odio mia Repubblica,/ Puttana dei signori dal sangue guastato $^{20} \gg$. Parole del genere, che suonano come una maledizione, non erano mai state scagliate contro i tribunali di Enver Hoxha. [...] Il poeta Xhagjika venne fucilato il 23 dicembre del 1963, all'età di 40 anni, insieme ad un gruppo di giovani artisti considerati "pericolosi" per il popolo. I suoi familiari attendono da mezzo secolo di sapere dove giacciono i resti del loro caro. Gli ex-membri del Pulitburo - che sono ancora vivi - e gli ex-ministri della cultura e i boia di Enver Hoxha sanno dove le guardie hanno gettato il corpo martoriato del poeta innocente, ma continuano a tacere.

Mentre il poeta ribelle Xhagjika andava ad affrontare il plotone di esecuzione della dittatura comunista, Kadaré scriveva una poesia dedicata al cane del confine - la bestia feroce rilasciata dalle guardie per mordere chi tentava di fuggire dai campi di internamento o dai lager. Kadaré, come scrive Kastriot Myftari «è l'unico poeta dei paesi comunisti dell'Europa dell'Est che abbia dedicato una poesia al cane del confine, a quell'epoca odiato da tutti, in quanto simbolo della dittatura e della repressione ${ }^{21}$ ». Ecco i suoi versi: «Respira velocemente./ Con gli occhi nervosi/ Si guarda intorno;/ Un collare verde/ Gli stringe la nuca muscolosa,/ Mentre cammina nel bosco con passi felpati.// Gli usignoli sui rami lo scrutano con ammirazione,/ I bei cerbiatti abbassano le corna in segno di rispetto/ E se i lupi lo incontrano per strada/ si fanno da parte per farlo passare ${ }^{22} 》$. Che cinismo ridicolo...

Vorrei riportare anche un'altra testimonianza come esempio di amore in mezzo a tutto quest'orrore. Si tratta di un altro poeta albanese, condannato a centoventi anni di carcere (!), che però, grazie al crollo della dittatura, ne ha scontati "solo" ventitré. È Gëzim Medolli, traduttore dei grandi scrittori della letteratura francese, oltre che dei poeti romantici inglesi. Oggi risiede in Grecia, ad Atene.

Medolli è stato arrestato per la prima volta nel 1973: era a casa e stava leggendo il Decameron quando la polizia segreta bussò alla sua porta per arrestarlo. Lo sbatterono in carcere con l'accusa di essere "un agitatore

20 Ibidem.

21 Kastriot Myftari, Gjyqi intelektual i Kadaresë, Buzuku, Prishtinë, Kosovë, 2008, p. 444.

22 Idem, p. 456. 
contro il potere del popolo", ma la verità è un'altra. Egli si era innamorato di una ragazza del suo villaggio che, per sua disgrazia, era la figlia del segretario del Partito Comunista locale. Questo fu considerato un grave disonore per la famiglia della fanciulla così il padre decise di vendicarsi separandoli per sempre: Gëzim venne incarcerato, mentre la ragazza si iscrisse all'università e si laureò in Giurisprudenza diventando giudice. In prigione Gëzim venne condannato più volte perché partecipò a delle rivolte nel carcere di Spaç dov'era rinchiuso: la sua pena arrivò a 120 anni! Egli non accettò alcuna collaborazione con il Sigurimi, che utilizzava varie forme per umiliare e calpestare l'onore dei carcerati, distruggerli come individui, riducendoli a dei relitti umani. Durante il terzo processo, e quindi la terza condanna, tra i membri della giuria del Tribunale del popolo vi era anche la sua amata, la sua 'campagnola', come lui la chiamava.

Dopo la condanna la ragazza non trovava pace, perché si era resa complice, in quanto giudice, di una mostruosa ingiustizia: aveva partecipato al processo e non si era opposta alla sua condanna.

Passano tanti anni e decide, infine, di scrivere una lettera al suo amato:

«Caro Gë...

Ti chiedo di perdonarmi per tutto quello che è accaduto tra noi. È per questo che oso scriverti, non per giustificarmi, ma per alleggerire la mia coscienza e per aver tradito l'unico mio amore che è vivo ancora nel profondo della mia anima sommersa dai tuoi ricordi. È ancora impressa nella mia memoria il momento in cui mi sono trovata di fronte a te durante le udienze tremende di un ingiusto processo. Dovevo agire con la ragione, invece di dar adito al mio lato più perfido di donna. In quel momento si risvegliarono in me ricordi incancellabili, quelli di un unico amore che invano avevo cercato di annegare dentro di me.

$\mathrm{Da}$ quel giorno ho perso la tranquillità e non riesco a trovare pace. Sono sconvolta. Nemmeno i baci dei miei figli, né i complimenti e le delicatezze del mio ufficiale [il marito], riescono a domare il mio tormento.

Ti amo, di un amore senza speranza! Immensamente, terribilmente, inutilmente! Su questa trinità di sentimenti mi abbandono per ritrovare il nostro amore bagnato di lacrime. Cerco di allontanare la tua immagine e di cancellarti dai miei sogni, ma, più passa il tempo, più diventi l'unico significato della mia esistenza, anche se non sono degna di te.

Ormai è tardi, troppo tardi, per correggere un errore e questa lettera è un altro errore, ma non potevo non scriverti. Sono una vigliacca, ma so che mi perdonerai e mi risponderai, dicendomi che sei sempre lo stesso.

Se è rimasta solo una lacrima nei miei occhi, sappi che è solo per te, per il tuo ricordo. 
Anche se non riesco a scrivere quel che sento in questo momento di buio, ti assicuro che sei l'unica gioia e, allo stesso tempo, l'unico tormento della mia anima.

Tua fino alla fine di ogni tempo

Y. M. ${ }^{23}$ ».

Gëzim lesse la lettera parecchie volte e le iniziali Y.M. attirarono la sua attenzione. In realtà lei avrebbe dovuto firmarsi Y.K., ma Gëzim capì che l'iniziale del cognome era Medolli, il suo. Così lei era diventata, di nascosto, la sua mancata consorte.

Gëzim prese un foglio e scrisse:

«Ti ho perdonato!

Che tu possa essere felice oltre ogni limite.

Tesoro.

G. M.».

Nel 1988, dopo vent'anni di carcere duro, Medolli tornerà in libertà e all'indomani dell'uscita dalla prigione verrà chiamato dal questore della sua città per essere ammonito con le parole seguenti: «Gëzim Medolli, se sbagli di nuovo ti attende il plotone!». Poteva fare solo il minatore. Nel 1991, dopo la caduta della dittatura comunista, Medolli decide di fuggire in Grecia, dove vive tuttora.

L. T.: Con Epicedio Hajdari non finisce il recupero della memoria storica collettiva del suo paese poiché ha già terminato un altro libro - che fa parte del suo progetto letterario - dal titolo Slogan dell'Albania di Enver Hoxha/ Parrulla enveriste. Il volume, che uscirà in Italia nel 2012, raccoglie 600 slogan che scandivano i tempi della dittatura insieme alle persecuzioni, alle condanne, alle prigionie. Per mezzo secolo essi hanno esercitato nella mente degli uomini e delle donne albanesi una sorte di terrore psicologico e di lavaggio del cervello. Lo scopo principale era diffondere le massime e le direttive del Partito Unico e tener viva la famigerata lotta di classe. Eccone qualche esempio:

«All'Albania finalmente è nato un figlio: il compagno Enver!» «L'opera più preziosa del Partito: la creazione dell'uomo nuovo!» «Trasformare moschee e chiese in palestre e case di cultura comunista!» «Gli insegnamenti del compagno Enver: più forti della bomba atomica!» «L'Albania è l'unico baluardo del comunismo nel mondo!» «L'Albania, l'unico paese al mondo senza tasse!»

23 G. Medolli, Globi në rrjetë, Eureka, Tiranë, 1994, pp. 98-99. 
«L'Albania, l'unico paese al mondo senza scioperi!»

«L'Albania, l'unico paese ateo al mondo!»

«Nel comunismo mangeremo con le posate d'oro»

Dal punto di vista letterario ecco alcuni slogan che condannano molti autori considerati 'pericolosi' per la nazione:

«Guerra alle influenze straniere e alle teorie reazionarie di Freud!»

«Abbasso gli scrittori traditori sovietici, cechi, bulgari, francesi, irlandesi e americani!»

«Abbasso i servi della degenerata società occidentale come Kafka, Joyce, Sartre, Kamy, Roger Garaudy, Natali Sarrot, Rob Grijene, Mishel Bytorit, Klod Simon, Solgenitsin, A. Kuznecov A. Demetjev, Tvardovskij, Xhon Hers e L. Andrejev!»

Ed ecco invece chi viene esaltato, perché la sua opera nutre il popolo, «affamato di letteratura del realismo socialista»: «L'opera di J. V. Stalin: sempre attuale!».

L'opera letteraria in bilingue di Gëzim Hajdari è quindi concepita come un grande poema epico a cui si aggiungono nel tempo i tasselli dolorosi della storia del suo paese, l'Albania, sino ad ora tenuti nascosti perché ingombranti. Ed è proprio dalla sua condizione di poeta migrante bilingue (italianoalbanese), condizione che egli considera certamente difficile e sofferta, ma anche privilegiata e necessaria, che egli riesce a compiere la propria missione per la madrepatria, per la letteratura, per la Storia umana ${ }^{24}$

24 Sulla storia dell'Albania e delle lettere albanesi durante il socialismo reale, cf. anche Nunzio Dell'Erba, La storia dell'Albania, Newton Compton, Roma, 1997; J. Bulo, L. Kokona, K. Bihiku, Historia e Letërsisë Shqiptare të Realizmit Socialist, Akademia e Shkencave e RPS të Shqipërisë. Istituti i Gjuhësisë e Letërsisë, Tiranë, 1983; Arsenij Roginskij, Quel che resta di un tiranno, in «La Repubblica», 5 marzo 2009. 